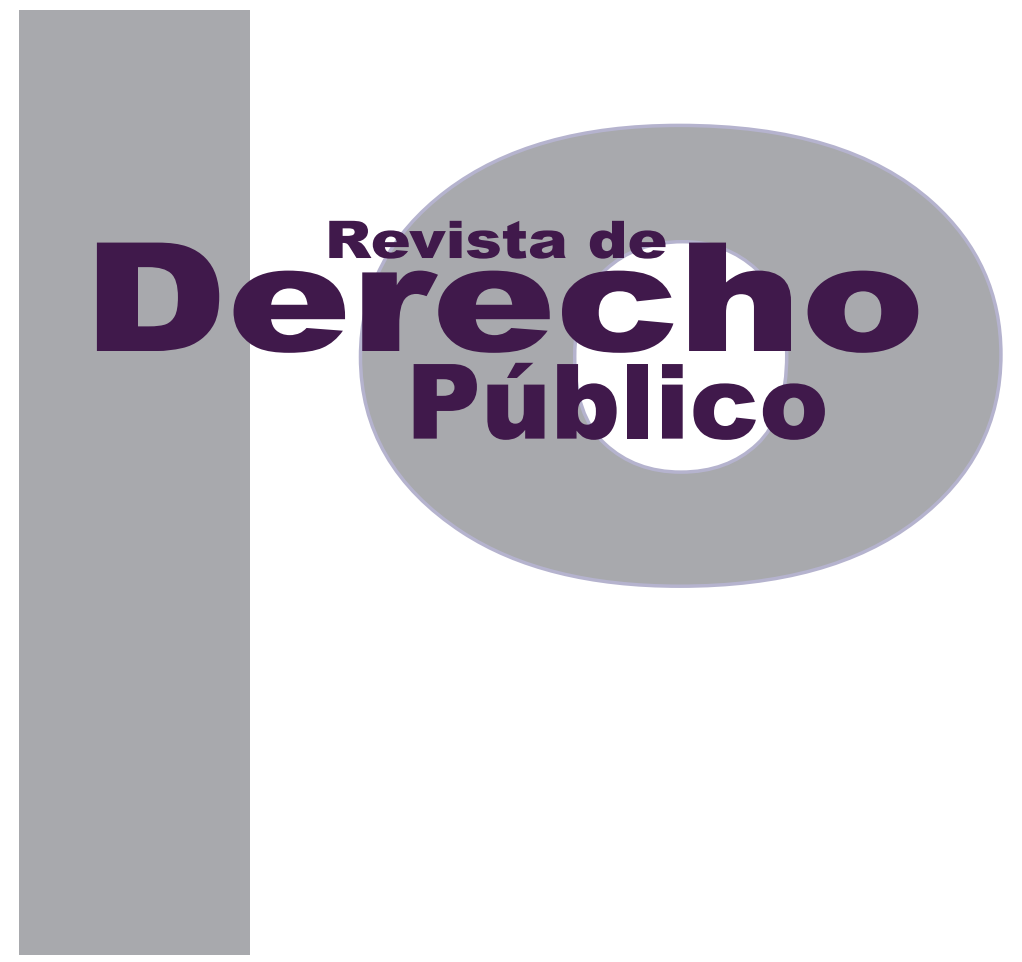

\title{
REACCIÓN DEL ESTADO COLOMBIANO FRENTE AL CARRUSEL DE LA CONTRATACIÓN EN BOGOTÁ: ¿EFICACIA O DISCURSO?
}

\author{
Diego Escallón Arango \\ Artículo de reflexión \\ Universidad de los Andes \\ Facultad de Derecho \\ Revista de Derecho Público N. ${ }^{\circ} 32$ \\ Enero - Junio de 2014. ISSN 1909-7778
}




\title{
Reacción del Estado colombiano frente al carrusel de la contratación en Bogotá: ¿eficacia o discurso?
}

\section{Resumen}

El carrusel de la contratación en Bogotá es el escándalo más grande de corrupción que se ha destapado en Colombia hasta el día de hoy. Como consecuencia de ese hecho, el Estado colombiano reaccionó impulsando la creación y modificación de normas jurídicas para combatirla, como la Ley 1474 de 2011 (Estatuto Anticorrupción). Este texto hace una evaluación y una comparación entre este caso y las medidas legislativas que se promulgaron para contrarrestar estos sucesos. Además, analiza la efectividad del Estatuto Anticorrupción desde el índice de percepción de corrupción de Transparencia Internacional y evalúa si efectivamente el derecho puede ser el medio para combatir esta práctica y erradicarla, o si el derecho, por su rigidez, no es suficiente para afrontar este flagelo social.

Palabras clave: Carrusel de la contratación, Estatuto Anticorrupción, eficacia, política pública, prevención, corrupción.

\section{Reaction of Colombian state with respect to the "hiring carousel" in Bogotá: Efficacy or speech?}

\begin{abstract}
The "hiring carousel" in Bogotá has been the biggest corruption scandal that has been dismantled in Colombia up to this day. Consequently, the Colombian state countered by promoting the creation and modification of legal rules as a way to defeat corruption, as illustrated, for instance, in the 1474 law of 2011 (Anti-Corruption Statute). This paper portrays an evaluation and a comparison between facts and legislative measures that were promoted to offset this corruption phenomenon. Furthermore, this piece analyzes the enforceability of the anti-corruption statute and evaluates if legislative measures are the correct path to defeat and eradicate corruption, or if law, because of its rigidity, is not enough to face this social calamity.
\end{abstract}

Keywords: Hiring carousel, Anti-corruption Statute, enforceability, public policy, prevention, corruption.

\section{Reação do Estado colombiano frenteao "carrossel da contratação" em Bogotá: eficácia ou discurso?}

\section{Resumo}

O carrossel da contratação em Bogotá foi o maior escândalo de corrupção que foi descoberto na Colômbia até hoje. Como consequência desse fato, o Estado colombiano reagiu impulsando a criação e modificação de normas jurídicas para combatê-la, como a Lei 1474 de 2011 (Estatuto Anticorrupção). Este texto faz uma avaliação e uma comparação entre este caso e as medidas legislativas que se promulgaram para contra restar estes acontecimentos. Além disso, analisa a efetividade do Estatuto Anticorrupção desde o índice de percepção de corrupção de Transparência Internacional e avalia se efetivamente o direito pode ser o meio para combater esta prática e erradicá-la, ou se o direito, por sua rigidez, não é suficiente para afrontar este flagelo social.

Palavras-chave: Carrossel da contratação, Estatuto Anticorrupção, eficácia, política pública, prevenção, corrupção. 


\section{SUMARIO}

Introducción - I. EL CARRUSEL DE LA CONTRATACIÓN EN BOGOTÁ: ¿QUÉ ES? - II. POSICIÓN Y REACCIÓN DEL ESTADO COLOMBIANO - A. El Plan Nacional de Desarrollo 2010-2014 y la lucha anticorrupción - B. Proyecto de Ley - nuevo Estatuto Anticorrupción - III. ¿LA SOLUCIÓN ES LEGISLAR? - A. Análisis de la necesidad de algunos artículos del Estatuto Anticorrupción para atacar la corrupción - B. Análisis de la efectividad del Estatuto Anticorrupción en la percepción de la corrupción y de la confianza del derecho como instrumento de cambio social, además de la nueva estrategia de política pública anticorrupción del Gobierno apoyada en el Plan Nacional de Desarrollo - IV. CONCLUSIONES - Bibliografía. 


\title{
Reacción del Estado colombiano frente al carrusel de la contratación en Bogotá: ¿eficacia o discurso? ${ }^{1}$
}

\author{
Diego Escallón Arango²
}

"La corrupción afecta a los pobres de manera desproporcionada porque desvía los fondos destinados originalmente al desarrollo, socava las capacidades de los gobiernos para ofrecer servicios básicos, alimenta las desigualdades y la injusticia, y disuade la inversión y la ayuda internacional".

Kofi Annan

Exsecretario general de las Naciones Unidas

\section{Introducción}

Casi todas las religiones y sistemas jurídicos han condenado la corrupción a través de la historia,

1 Cómo citar este artículo: Escallón A., D. (Junio, 2014). Reacción del Estado colombiano frente al carrusel de la contratación en Bogotá: ¿eficacia o discurso? Revista de Derecho Público, 32

2 Candidato a grado de abogado con opción en Administración de la Universidad de los Andes. Asesor del director del Sistema Nacional de Bienestar Familiar en el Instituto Colombiano de Bienestar Familiar y tutor del curso Hermenéutica Jurídica de la Facultad de Derecho de la Universidad de los Andes. Correos: diego.escallon@gmail.com; d.escallon2702@uniandes.edu.co

Agradezco especialmente a Joaquín Vélez por su acompañamiento, apoyo y generosidad tanto en la preparación de este escrito como a lo largo de mi carrera. Además agradezco a Carlos Fernando Galán, Diana Mejía y Rafael Merchán por su tiempo y colaboración para el desarrollo de esta investigación. sin embargo no se ha logrado combatir con eficacia (Pieth, 2011). La corrupción hace que el Estado y las instituciones democráticas queden en tela de juicio, ya que "afecta la imagen del Estado pues este pierde legitimidad, en la medida [que] no cumple con sus fines y se distorsiona la efectividad de las políticas públicas implementadas por el mismo" (Vélez, 2013).

La corrupción se ha definido de varias formas y en diversos sentidos, por ejemplo, se ha dicho que: "el acto corrupto es la defraudación privada del sector público. El funcionario [...] distorsiona su investidura, para actuar con un interés privado" (Nas, Price y Weber, 1986, citados en Vásquez Caro, 1994, pág. 317). Todas las definiciones tienen un elemento en común: siempre se atenta contra el interés público (PNUD, 2011). Este flagelo corroe la dignidad de los ciudadanos y los contamina, deteriora la convivencia social, afecta los servicios públicos y la vida de generaciones actuales y futuras (Amarribo, 2010). Sin embargo, luchar contra este mal es muy complejo debido a que se presenta mediante 
prácticas esporádicas o de manera sistémica, en las cuales se captura al Estado y hay una cooptación del servicio público para un beneficio individual (Maldonado Copello, 2011).

Colombia no es ajena a este fenómeno. Mientras Antanas Mockus, durante su candidatura a la Presidencia de la República en el año 2010, decía: "Ios recursos públicos son sagrados", el Distrito Capital, y el país entero, se preparaban para conocer uno de los escándalos de corrupción más grandes de nuestra historia: el carrusel de la contratación. Este hecho fue destapado por el exconcejal Carlos Fernando Galán y su equipo, entre los años 2009 y 2010. Su importancia justifica este escrito.

En este texto se analiza el carrusel de la contratación y, con base en ese escándalo, se evalúan las reacciones legislativas del Estado colombiano para combatir la corrupción, y las normas creadas como consecuencia de este caso específico. Al efecto, se comparan los hechos del carrusel de la contratación con la Ley 1474 de 2011 (Estatuto Anticorrupción), con el fin de revisar su efectividad, y evaluar si realmente el derecho puede ser el medio para combatir y erradicar la corrupción o si es insuficiente ante la velocidad y capacidad de adaptación de los corruptos, todo esto a la luz de la formulación de políticas públicas y de las nuevas miradas políticas por las que ha optado el Gobierno Nacional para afrontar este flagelo social. Con base en lo anterior, se argumenta que la corrupción es un acto tan complejo que no puede ser combatido solamente desde el derecho, y menos legislando con posterioridad a los hechos, sino que debe ser analizada y atacada desde varias áreas del conocimiento buscando su prevención. Además, debe estar acompañado de medidas de control y culturización de la ciudadanía para disminuir la legitimidad de ese tipo de actuaciones.

Mientras los corruptos se acomodan rápidamente a las nuevas reglas anticorrupción, la rigidez de las normas jurídicas y el procedimiento que debe surtirse para modificarlas dificulta la adaptación del sistema a los métodos de corrupción que van surgiendo. Por eso es importante que el discurso contra la corrupción se dé desde el "por qué" del incumplimiento y no desde el "cómo" se incumple, pues el síndrome legislati$v^{3}{ }^{3}$, está visto, no ha logrado erradicar la cultura de la corrupción en el país. Hasta ahora el Estado despertó y se dio cuenta de esto. En otras palabras, en este escrito se argumenta que la corrupción no se debe combatir solo desde el derecho legislado, sino que se deben adoptar políticas públicas de prevención, teniendo en cuenta otras áreas del conocimiento, y enfocarse en el porqué de este fenómeno.

Con tal propósito, en el primer capítulo se hace una breve descripción de los principales hechos del carrusel de la contratación, los implicados, los métodos usados por ellos y el proceso de desmantelamiento, con base en noticias de prensa, ${ }^{4}$ algunos hechos confirmados por el

3 El síndrome legislativo entendido como la creencia del Legislador colombiano de que los cambios sociales y la resolución de los problemas sociales está en el derecho. Para eso todas las problemáticas se intentan resolver modificando o construyendo nuevas leyes.

4 Los medios de comunicación jugaron un rol central en cuanto a instalar la corrupción en la agenda pública y aportar la información inicial para la toma de decisiones en este sentido. (Banco Interamericano de Desarrollo, 2012) 
Consejo de Estado y el relato de las personas que fueron pieza clave en el desarrollo de esta investigación: el exconcejal Carlos Fernando Galán ${ }^{5}$ y Diana Mejía, ${ }^{6}$ su asesora.

En el segundo capítulo se describen las reacciones normativas del Estado colombiano frente a este fenómeno, que pueden resumirse principalmente en dos: i) el Plan Nacional de DesarroIlo 2010-2014 (Ley 1450 de 2011) y ii) el nuevo Estatuto Anticorrupción (Ley 1474 de 2011). En ese capítulo describiré las principales normas que, en comparación con los hechos del carrusel de contratación, buscan contrarrestar los métodos y acciones que dieron lugar al carrusel. Debo advertir que en este texto solo se analiza el Estatuto Anticorrupción.

En el último capítulo se evalúa si la solución para la corrupción está en las leyes y si la reacción del Estado colombiano frente al carrusel fue la correcta, o si debido a la magnitud del problema es un hecho que debe controlarse a través de políticas públicas sólidas que permitan atacar sus causas estructurales y no desgastar los esfuerzos del Estado previniendo la repetición de conductas jurídicas ya ocurridas. Para esto, la evaluación de la reacción del Estado se dividió en dos: i) análisis de la necesidad de algunos artículos del Estatuto Anticorrupción para atacar la corrupción, y ii) análisis de

5 Político colombiano que se desempeñó como concejal de Bogotá entre 2008 y 2011 y candidato a Alcalde de la capital en las elecciones de 2011. Actualmente es senador de la República y director del partido político Cambio Radical.

6 Exasesora de Carlos Fernando Galán durante los años 2009-2010. Actualmente trabaja en el Observatorio de Transparencia y Corrupción de la Presidencia de la República. la efectividad del Estatuto Anticorrupción en la percepción del fenómeno y de la confianza en el derecho como instrumento de cambio social, además de la nueva estrategia de política pública anticorrupción del Gobierno, apoyada en el Plan Nacional de Desarrollo. La revisión de la eficacia del Estatuto Anticorrupción se apoyó en un índice altamente legitimado por instituciones públicas y privadas a nivel internacional: el índice de percepción de la corrupción (IPC) que publica Transparencia Internacional desde 1993. Para la comparación que aquí se aborda, los años parámetro fueron los del 2008 al 2013. Este análisis permite observar si esa norma jurídica ha generado alguna variación en el índice de corrupción y si, tomando dicho índice como base, puede decirse que fue efectiva o que se redujo a un simple populismo legislativo y que demostró que el Estado debía cambiar su estrategia para confrontar la corrupción.

Antes de empezar el desarrollo de los aspectos anotados debo advertir que la creencia en el legalismo francés y el populismo lingüístico ${ }^{7}$ me llevó a escribir el texto pensando en llegar tanto a abogados como a personas de otras áreas, por eso el lenguaje es común y no jurídico.

\section{EL CARRUSEL DE LA CONTRATACIÓN EN BOGOTÁ: ¿QUÉ ES?}

En este capítulo se describen los hechos principales del carrusel de la contratación y su de-

El profesor López Medina llama populismo lingüístico a la labor del legislador francés de dirigir el derecho a todos los ciudadanos del territorio, pues este no puede ser un sistema profesional que solo aquellos educados en él logren descifrar (2008, págs. 10-13) 
sarrollo. Como este fue un fenómeno tan complejo acá solo se refieren los hechos principales que se destaparon entre los años 2009 y 2010, pues muchos aún están en investigación y la información, por lo tanto, es confidencial. Las fuentes que respaldan esta parte son informes de prensa, sentencias judiciales y las entrevistas realizadas a Carlos Fernando Galán y a Diana Mejía. Empieza la descripción:

Miguel Eduardo Nule Velilla -hijo mayor del político del departamento de Sucre, Miguel Nule Amín, e ingeniero civil de la Universidad de los Andes-, junto con su hermano Manuel Francisco Nule Velilla y su primo hermano Guido Alberto Nule Marino eran los dueños del Grupo Nule, el cual estaba compuesto por quince empresas. Entre las más importantes figuraban: MNV S. A. (hoy en liquidación); Gas Kpital Gr S. A. (hoy en liquidación); Kpital Energy S. A.; Translogistic S. A.; Compañía Energética del Tolima S. A.; Enertolima Inversiones S. A. ESP (Consejo de Estado. Sala de lo Contencioso Administrativo. Sec. Quinta. C. P: Susana Buitrago Valencia. 25 de enero de 2013. Radicado n. 11001-03-15000-2012-02311-00)

En 1998, estos empresarios sucreños se trasladaron a Bogotá y resultaron adjudicatarios de sus primeros contratos con el Instituto Nacional de Vías (Invías) y con el Distrito Capital. Los Nule, con sus empresas, ejecutaron contratos para el Estado colombiano y "sus inversiones traspasaron las fronteras, además de varios países de América Latina desarrollaron proyectos en España, y en busca de recursos llegaron a China y Dubái" (Revista Semana, 2010).
Las empresas MNV S. A. y Gas Kpital GR S. A., fueron las dos más importantes del Grupo Nule. La primera de estas, asociada a otras sociedades comerciales, fue favorecida con varias obras de infraestructura en Colombia; algunas de las más importantes y que los posicionaron como los grandes constructores del país fueron: la Concesión de la doble calzada Bogotá-Girardot (Contrato Instituto Nacional de Concesiones [INCO], hoy Agencia Nacional de Infraestructura [ANI], n. ${ }^{\circ}$ GG 040 de 2004) y la tercera fase de Transmilenio para la construcción de la Calle 26 en Bogotá (Contrato Instituto de Desarrollo Urbano de Bogotá D. C. [IDU] 137-2007).

De acuerdo con Diana Mejía, para el año 2009 los Nule tenían a su cargo millonarios contratos con el Estado para la construcción de obras de infraestructura, prestación de servicios de gas, de energía, de agua y el suministro de bienestarina (Mejía, 2013). Facturaban cerca de 200 millones de dólares al año y empleaban, directa e indirectamente, cerca de 15 mil personas (Revista Semana, 2010).

Por todo lo anterior, los Nule eran reconocidos como la nueva generación de grandes constructores del país y con excelentes proyecciones a futuro, puesto que eran jóvenes, exitosos y multimillonarios. La historia real no es tan optimista.

Relata Carlos Fernando Galán, que a mediados del año 2009 un subcontratista al que le habían incumplido un pago de 215 millones de pesos, de manera anónima avisó a su oficina que el IDU iba a hacer una modificación al Contrato IDU137-2007, que consistía en permitirle al contra- 
tista -los Nule - realizar una "actualización en los diseños". Este hecho llamó su atención porque, según señala, "el Distrito iba a pagar nuevamente los diseños de la obra y se reducían las condiciones para el pago de anticipos porque se reducían las condiciones para pagarles" (Galán, 2013); por esa razón él y su equipo comenzaron la investigación que destapó uno de los fenómenos de corrupción más grandes de la historia de Colombia. Posteriormente, una fuente de la interventoría de ese contrato dijo a la oficina del concejal Galán, que el carrusel y la Fase III de Transmilenio estaban conectados.

El concejal Galán, con el apoyo de su hermano, el senador Juan Manuel Galán, solicitó al Distrito la información sobre el proceso contractual de la calle 26; la contestación fueron ocho cajas con documentos que por su volumen buscaban desincentivar la investigación. A pesar de ello, el personal de la oficina del concejal revisó uno a uno todos los documentos y de ahí surgieron los primeros hallazgos e irregularidades con los Nule (Galán, 2013).

Con base en estos datos más la ayuda de fuentes anónimas que le brindaron información, el concejal destapó algunas de las maniobras de corrupción del Grupo Nule con Emilito Tapia, Julio Gómez y Álvaro Dávila (algunos de los contratistas inmersos, hasta donde se sabe, en el carrusel). Pero la opinión pública solo concentró su atención en estos hechos hasta el 25 de junio de 2010, cuando Caracol Radio reveló una conversación entre el excongresista Germán Olano y Miguel Nule Velilla en la que involucraban al Alcalde Mayor de Bogotá, Samuel Moreno, a su hermano el exsenador Iván Moreno Rojas y al contralor de Bogotá Miguel Ángel Moralesrussi, en la presunta negociación de comisiones para la adjudicación de los megacontratos del Distrito (Consejo de Estado, Sala Plena de lo Contencioso Administrativo, C. P: Gustavo Eduardo Gómez Aranguren, Radicación n. 11001-0315-000-2011-00125-00).

La investigación de Galán encontró que nueve licitaciones entre las que estaban la rehabilitación de la malla vial, la fase III de Transmilenio y otros grandes proyectos de la capital, habían sido repartidas entre estos contratistas, a través de la entrega de comisiones ilegales a Samuel Moreno Rojas, Iván Moreno Rojas y otros funcionarios del Estado. De acuerdo con las investigaciones que ha adelantado la Fiscalía y los órganos de control, el abogado Álvaro Dávila fue el intermediario entre los Nule y los hermanos Moreno (EI Tiempo, 2011). El fiscal delegado ante la Corte Suprema de Justicia encargado del caso, Juan Valbuena, afirmó a la revista Semana (2013): “desde diciembre del 2007 y hasta junio del 2010, Samuel Moreno, su hermano Iván, el abogado Álvaro Dávila y los empresarios Emilio Tapia y Julio Gómez se pusieron de acuerdo para cometer delitos contra la administración pública".

A mediados del 2012, Emilio Tapia lo confesó todo e hizo un preacuerdo con la Fiscalía para testificar en contra de los involucrados y disminuir su condena. En la declaración manifestó que su labor en el carrusel era coordinar a los contratistas y recaudar la plata para las comisiones de los hermanos Moreno (Revista Sema- 
na, 2012). Y según el mapa que hizo a la Fiscalía, las entidades que se vieron implicadas en temas de corrupción en la contratación pública fueron las siguientes: Empresa de Acueducto y Alcantarillado de Bogotá, Caja de Vivienda Popular, Fondo de Vigilancia y Seguridad, Unidad Administrativa Especial de Servicios Públicos UAESP-, Secretaría de Salud, Terminal de Transporte, la recolección del Sistema Integrado de Transporte Público, algunas alcaldías locales y comedores comunitarios (Revista Semana, 2012). La gran mayoría de la contratación del Distrito Capital estaba concentrada en las empresas del Grupo Nule.

Adicionalmente, en general las denuncias vincuIaron a Liliana Pardo, exdirectora del IDU; a los exconcejales Hipólito Moreno, Andrés Camacho Casado, Jorge Durán Silva, Ernesto Salamanca, Alfonso Parada, Wilson Duarte y Javier Palacio; a Inocencio Meléndez, ${ }^{8}$ exdirector jurídico del IDU; a Edmundo del Castillo, exsecretario jurídico de la Presidencia; a Francisco Rojas Birry, personero de Bogotá y a otros servidores públicos de gran importancia en el Estado colombiano (Mejía, 2013). Estas personas aparecieron en las denuncias porque, presuntamente, habían participado en la repartición y concentración ilícita de contratos en Bogotá.

Para esta época, marzo de 2010, las dos empresas más importantes de los Nule estaban cerca

8 Su participación se asoció a la suscripción de un contrato con Urías Torres, un abogado a quien se le contrató para defender el Acuerdo 180 (contribución de valorización de la capital) en el que la comisión de éxito era el $0.3 \%$ de todo el recaudo de la ciudad por esa contribución. A los dos días de firmar el contrato lo cedió a la esposa del magistrado del Tribunal Administrativo de Bogotá, que había tenido el caso inicialmente (Galán, 2013). de declararse en insolvencia y, sumado a esto, se hacían evidentes los incumplimientos en la ejecución de los contratos que tenían con el Estado. El gran imperio que habían construido los Nule se derrumbó por el indebido manejo que le dieron a los anticipos ${ }^{9}$ de los contratos que les habían sido adjudicados. Sus problemas financieros los llevaron a usar los anticipos de unos contratos para cubrir los incumplimientos de otros, creando el círculo vicioso en que vivieron hasta que su músculo financiero no aguantó más. El punto final lo puso la pérdida de la adjudicación de la Concesión Vial Ruta del Sol, porque al no disponer de este anticipo el círculo vicioso se rompió, quedaron insolventes ${ }^{10}$ y sobrevino el colapso financiero (Mejía, 2013).

De acuerdo con la información publicada en la prensa, las comisiones ilegales funcionaban así: los contratistas negociaban entre ellos qué contrato de la Alcaldía de Bogotá quería cada uno y, de acuerdo con su decisión, lo aseguraban a través de garantizarle un porcentaje a los hermanos Moreno Rojas (Revista Semana, 2013). Además de las dádivas ilegales, Julio Gómez había constituido una red de fundaciones para tener como fachada la contratación y desviar la atención de los entes de control. Estas

9 Es el dinero anticipado que entrega la entidad contratante al contratista para el cubrimiento de los costos en que este debe incurrir para iniciar la ejecución del contrato. Sigue perteneciendo a la entidad estatal por lo que debe destinarse exclusivamente al contrato para el cual fue entregado (DNP, 2010).

10 El 10 de septiembre de 2010 la Superintendencia de Sociedades (2010), por medio de un auto, ordenó la liquidación judicial de todo el Grupo Nule, sustentada en que este actuaba como tal sin haberse declarado oficialmente, tendría irregularidades contables y financieras y, adicionalmente, estaba en cesación de pagos desde hacía varios meses. 
fundaciones fueron adjudicatarias de contratos millonarios con el Distrito (Galán, 2013). Según las investigaciones y la información revelada así era su método de acción (Caracol Radio, 2011).

Aunque aún no ha sido confirmado, las investigaciones señalan que varios contratistas de los implicados en el carrusel habrían constituido un fondo privado para financiar la campaña de Samuel Moreno a la Alcaldía de Bogotá.

Adicionalmente, en octubre del 2010, Gustavo Petro, en ese entonces senador, junto con el concejal Carlos Vicente de Roux y el senador Luis Carlos Avellaneda presentaron otro informe en el que confirmaban las denuncias contra el exalcalde Moreno y su hermano Iván, además de otros altos funcionarios implicados.

Como se registra en el acta de aceptación de cargos que se produjo en el proceso en contra del exrepresentante a la Cámara Germán Olano, para la adjudicación del contrato IDU-137-2007 (Transmilenio calle 26) Miguel Nule Velilla acordó con Germán Olano y Julio Gómez el pago de 3.500 millones de pesos, a razón del 50\% para cada uno, es decir, 1.750 millones para el excongresista y 1.750 millones para Julio Gómez, arreglo que incluía también la entrega de una parte de esta suma a los funcionarios del IDu encargados de la adjudicación del contrato: Liliana Pardo Gaona e Inocencio Meléndez Julio. (Corte Suprema de Justicia, Sala de Casación Penal, M. P.: Fernando Alberto Castro Caballero, Acta n. ${ }^{\circ} 363$ del 27 de septiembre de 2012).

Además de todo lo anterior, se empezó a hacer evidente que los contratos habían sido adjudi- cados a personas que no estaban calificadas para ejecutarlos. Esto se puede comprobar, entre otros, con el contrato de la Concesión de la Autopista Bogotá-Girardot, obra que ha tenido varios retrasos por algunos problemas que la gerente técnica de la empresa concesionaria, Ana Milena Medina, resume en carta enviada al INCO -hoy ANI-:

El problema principal de la Concesión está asociado a la severa crisis que afecta al Grupo Nule, en la medida en que por efectos de la situación financiera que se venía gestando desde hace varios meses, los contratos asignados al Consorcio Bogotá Fusa (CBF), la compañía del Grupo Nule responsable de la construcción del $50 \%$ de las obras a ser realizadas por la concesión, presentan sustanciales atrasos. (Medina, 2010, pág. 2).

Los socios de la Concesión Autopista BogotáGirardot son: MNV S. A. (hoy en liquidación), Gas Kpital GR S. A. (hoy en liquidación), Álvarez y Collins S. A., Vergel y Castellanos Ingenieros Asociados VyC Ltda. y Alejandro Char Chaljub. En esta Concesión las empresas de los Nule, además de socias eran subcontratistas de las obras. En este solo consorcio los Nule dejaron de amortizar anticipos por aproximadamente 68 mil millones de pesos y se quedaron con ese dinero ilegalmente, puesto que debía ser destinado a la construcción de la vía (BRC Investor Services S. A., 2011). En otros contratos, de acuerdo con un estimativo de anticipos hecho por El Tiempo (2010), entre los años 2003 y 2010 los Nule dejaron de amortizar anticipos por cerca de 141 mil millones de pesos. 
Además, las investigaciones señalan que la corrupción en la contratación pública por parte del Grupo Nule y otros contratistas no se presentó solo en Bogotá, sino que las comisiones ilegales pagadas a servidores públicos para acceder a millonarios contratos se dieron en varias capitales y municipios del país.

A la fecha (2014), los jueces penales han encontrado culpables a Inocencio Meléndez, Miguel Nule, Manuel Nule, Guido Nule y Mauricio Galofre por el delito de peculado por apropiación, y al exrepresentante a la Cámara Germán Olano por el delito de enriquecimiento ilícito y tráfico de influencias. Este aceptó los cargos y se acogió al beneficio de sentencia anticipada. Frente a los demás involucrados actualmente se adelantan los juicios penales en su contra.

\section{POSICIÓN Y REACCIÓN DEL ESTADO COLOMBIANO}

En este capítulo se describe la posición y reacción del Estado colombiano con respecto a los hechos narrados en el capítulo anterior, que fueron conocidos entre los años 2009 y 2010. Como antecedentes se mencionan brevemente las medidas tomadas por el Estado con anterioridad para afrontar la corrupción en general. Como ya se aclaró, este capítulo es informativo y descriptivo, pues el análisis sobre la efectividad de la reacción del Gobierno Nacional se analiza en el siguiente capítulo.

Desde hace varios años el Estado colombiano ha buscado disminuir la corrupción en el país. El
Congreso de la República promulgó la Ley 190 de 1995 "Por la cual se dictan normas tendientes a preservar la moralidad en la administración pública y se fijan disposiciones con el fin de erradicar la corrupción administrativa", norma conocida como el Estatuto Anticorrupción. Dos años después, ante la gravedad de las consecuencias de las acciones corruptas, Colombia ratificó la Convención Interamericana contra la Corrupción, por medio de la Ley 412 de 1997. Posteriormente, mediante la Ley 970 de 2005 el país aprobó la Convención de las Naciones Unidas contra la corrupción, en donde en el preámbulo se expresa la preocupación de los Estados miembros por "los casos de corrupción que entrañan vastas cantidades de activos, los cuales pueden constituir una proporción importante de los recursos de los Estados, y que amenazan la estabilidad política y el desarrollo sostenible de esos Estados" (párrafo 3).

En este marco de normas anticorrupción, compromisos internacionales vigentes y un Estatuto de Contratación Pública bastante riguroso, el carrusel de la contratación se desarrolló con tranquilidad y demostró la debilidad de las instituciones públicas colombianas para combatir este flagelo. El cohecho, el tráfico de influencias y el nepotismo continuaron primando sobre la gobernabilidad pública.

Por si fuera poco el escándalo en los medios de comunicación por el carrusel de la contratación, por la misma época el Barómetro Global de Corrupción de Transparencia Internacional ubicó a Colombia en el puesto 74 entre 189 países evaluados (Ministerio del Interior y de Justicia, 
2011), con un puntaje de 3.5 donde 1 es para nada corrupto y 5 es extremadamente corrupto (Transparencia Internacional, 2009). En ese contexto, el Gobierno Nacional se vio en la obligación de tomar medidas diferentes a las acciones penales, fiscales y disciplinarias en contra de los implicados en el carrusel de la contratación para disminuir la percepción y los actos de corrupción. La primera fue la inclusión en el Plan Nacional de Desarrollo 2010-2014 de la propuesta de lucha contra la corrupción. Como refuerzo de la anterior, a través del entonces Ministerio del Interior y de Justicia presentó ante el Congreso de la República el Proyecto de Ley 142 de 2010, orientado a fortalecer los mecanismos de prevención, investigación y sanción de actos de corrupción y la efectividad del control de la gestión pública, iniciativa que una vez aprobada se convirtió en la Ley 1474 de 2011, más conocida como nuevo Estatuto Anticorrupción.

\section{A. El Plan Nacional de Desarrollo 2010- 2014 y la lucha anticorrupción}

El Plan Nacional de Desarrollo "es la base de las políticas gubernamentales formuladas por el Presidente de la República, a través de su equipo de Gobierno y plasma los temas y propuestas expuestas a los colombianos durante su campaña electoral" (DNP, 2010). En ese sentido, contiene las directrices de gobernanza que este debe seguir durante el periodo para el cual fue elegido.

El Plan Nacional de Desarrollo 2010-2014 fue aprobado mediante la Ley 1450 de 2011. En este se incluyó como eje transversal el "Buen
Gobierno como principio rector en la ejecución de las políticas públicas, y en la relación entre la Administración y el ciudadano". A su vez, dentro del Buen Gobierno se consagra la lucha contra la corrupción y algunas estrategias para su control.

Debe rescatarse que es la primera vez, en los últimos dieciséis años, que en un Plan Nacional de Desarrollo se contemplan medidas dirigidas hacia la prevención de la corrupción; sin embargo, en la práctica solo hasta el 2013 se les dio algún desarrollo, como se verá más adelante.

\section{B. Proyecto de Ley y nuevo Estatuto Anticorrupción}

La segunda medida, y la principal, fue el Proyecto de Ley 142 de 2010 presentado al Congreso por el entonces ministro del Interior y de Justicia, Germán Vargas Lleras. Este proyecto fue aprobado y se convirtió en la Ley 1474 de 2011 (en adelante Ley 1474) o nuevo Estatuto Anticorrupción.

En la exposición de motivos del Proyecto de Ley, publicada en la Gaceta del Congreso 607 de 2010, se describe la importancia de atacar la corrupción como fenómeno dinámico cuyas manifestaciones varían con el transcurso del tiempo y afectan la institucionalidad y la gobernabilidad pública. Precisamente, ese dinamismo descrito en la exposición de motivos fue el que impulsó al Gobierno a reaccionar ante las circunstancias que vivía el país -el carrusel de la contratación y el índice de percepción de la corrupción que venía registrando Colombia en el Barómetro Global de Corrupción-. Según la 
exposición de motivos: "el presente proyecto de ley busca introducir nuevas disposiciones que se ajusten a las necesidades actuales que la lucha contra la corrupción exige, propendiendo subsanar e integrar aquellos aspectos en los cuales se requiere una acción contundente."

La modernización propuesta por el Gobierno para ajustar la legislación a la situación del país encontró apoyo en el Congreso, y de esta manera la nueva norma pretendió desarrollar aspectos como el buen gobierno, la transparencia, la participación ciudadana y la disminución de beneficios penales a los culpables de los delitos contra la administración pública (Instituto de Ciencia Política Hernán Echavarría Olózaga, 2011). Adicionalmente, busca desincentivar los beneficios de los agentes inmersos en la corrupción, como lo dijo el exministro Vargas Lleras en la presentación de la Ley 1474:

La corrupción se presenta porque existe un mercado en el que se puede desarrollar. Existen agentes demandantes y oferentes de corrupción que obtienen ganancias por las transacciones de las que participan. Las políticas anticorrupción deben incorporar medidas orientadas a eliminar la oferta de corrupción por parte de los agentes públicos y la demanda privada (Ministerio del Interior, 2011, pág. 7).

La Ley 1474 está compuesta por 135 artículos, divididos en diez capítulos: medidas administrativas (capítulo 1), penales (capítulo 2), disciplinarias (capítulo 3), de regulación del lobby o cabildeo (capítulo 4), organismos especiales (capítulo 5), políticas institucionales y pedagógicas (capítulo 6), de prevención (capítulo 7), de eficiencia y eficacia del control fiscal (capítulo 8), regulación de las oficinas de representación (capítulo 9) y vigencia (capítulo 10).

Para el análisis de los hechos relacionados con el carrusel de la contratación se acudirá principalmente al capítulo 7 , concretamente a las disposiciones para prevenir y combatir la corrupción en la contratación pública, a saber:

i) Expansión de la responsabilidad fiscal y disciplinaria de los consultores, asesores externos e interventores por daños o perjuicios a las entidades, derivados de la celebración y ejecución de los contratos en los que tengan relación (artículo 82).

ii) Expansión de la obligación de los interventores y supervisores de los contratos para ejercer vigilancia sobre las posibles actuaciones de corrupción que puedan evidenciar, y el correlativo deber de informarlo a las autoridades de control (artículo 84).

iii) Inhabilidad para los contratistas que sean sancionados durante la ejecución de contratos, incluyendo a los socios de las sociedades de personas (artículo 90).

iv) En los contratos de obra, concesión, salud o los que sean adjudicados por licitación pública, el contratista adjudicatario debe constituir una fiducia o un patrimonio autónomo irrevocable al que trasladará los anticipos entregados por el Estado, con el fin de garantizar su correcta inversión (artículo 91). 
vi) Adicionalmente, se consagraron ciertas normas que excluyen los beneficios penales en los delitos contra la administración pública (artículo 13), se amplió el término de prescripción de la acción penal (artículo 14), se elevó a delito los acuerdos restrictivos de competencia cuando en un proceso de licitación pública, subasta pública, selección abreviada o concurso se concierte con otro oferente con el fin de alterar ilícitamente el procedimiento contractual (artículo 27), entre otras disposiciones que crearon nuevos delitos y aumentaron las penas por corrupción.

Por último, se destaca el artículo 79 que no está relacionado con el manejo de los recursos de los contratos sino con la pedagogía de las competencias ciudadanas, mandato que exige "que los establecimientos educativos de educación básica y media incluyan en su proyecto educativo institucional programas destinados a fomentar la cultura de la legalidad y el cuidado de los bienes comunes" (Lombana y Castro, 2012). Esta norma está relacionada, como se verá más adelante, con la nueva visión del Gobierno frente al manejo de la corrupción: propender por la prevención antes que por el control posterior.

En conclusión, la reacción del Estado colombiano frente al carrusel de la contratación tuvo dos expresiones: una legal, al consagrar normas que bloquean la posibilidad de usar indebidamente los anticipos de los contratos, agregar nuevas causales de inhabilidad para los contratistas, excluir los beneficios penales por delitos cometidos contra la administración pública y, en general, desincentivar a los servidores públicos $y$ al sector privado a caer en actuaciones delictivas de este tipo; y otra pedagógica, orientada a crear, desde las aulas escolares, una cultura de respeto por los recursos públicos.

\section{III. ¿LA SOLUCIÓN ES LEGISLAR?}

En este capítulo se analizan las medidas tomadas por el Estado colombiano como reacción al carrusel de la contratación y al contexto político y social en materia de corrupción que vivía el país entre los años 2009 y 2010.

Antes de desarrollarlo conviene recapitular lo dicho hasta aquí: entre los años 2009 y 2010 salió a la luz pública un caso de corrupción sin precedentes en el país, protagonizado por varios contratistas y funcionarios públicos de altos cargos. Estos hechos evidenciaron principalmente un indebido manejo de los anticipos sobre contratos del Estado, incumplimientos contractuales como consecuencia del manejo ilícito de los dineros recibidos como anticipo y pagos ilegales a funcionarios y políticos de importancia en las instituciones nacionales, a cambio de contratos adjudicados violando los requisitos legales. A lo anterior el Gobierno reaccionó incluyendo en el Plan Nacional de Desarrollo 2010-2014 (Ley 1450 de 2011) un capítulo relacionado con la lucha contra la corrupción; presentando el proyecto de ley que luego se convirtió en la Ley 1474, con el fin de atacar varios frentes de corrupción y, principalmente, regular el manejo de anticipos por parte de los contratistas del Estado, aumentar las inhabilidades de contratación 
con entidades públicas y elevar la responsabilidad de las interventorías.

Ahora bien, el análisis de la reacción del Estado se ocupa de dos aspectos: i) la necesidad de algunos artículos del Estatuto Anticorrupción para atacar este problema, y ii) la efectividad del Estatuto Anticorrupción en la percepción de la corrupción y de la confianza en el derecho como instrumento de cambio social, además de la estrategia de política pública anticorrupción del Gobierno actual, plasmada en el Plan Nacional de Desarrollo 2010-2014.

\section{A. Análisis de la necesidad de algunos artículos del Estatuto Anticorrupción para atacar la corrupción}

Al revisar los hechos del carrusel de la contratación a la luz de las normas descritas con anterioridad, es posible concluir que tales disposiciones se promulgaron con base en este caso y que buscaban prevenir y combatir contravenciones similares.

Antes de la expedición de la Ley 1474 ya había algunas normas que, en teoría, estaban dirigidas a prevenir que hechos como el carrusel de la contratación pudieran pasar; por citar algunos ejemplos: artículos 8, 9, 40 y 53 de la Ley 80 de 1993 y los artículos 7 y 18 de la Ley 1150 de 2011. Sin embargo, estas no fueron suficientes y el Gobierno se vio en la necesidad de presentar el proyecto de ley que, luego del trámite legislativo, dio vida al nuevo Estatuto Anticorrupción.
Si se analizan los artículos de la Ley 1474 parafraseados con anterioridad, es claro que estaban inspirados en el carrusel de la contratación. Veamos:

1. Aumentar la responsabilidad de los interventores y el deber de denunciar hechos de corrupción. Aunque el artículo 53 de la Ley 80 de 1993 ya definía el grado de responsabilidad de los interventores, si ellos y los supervisores hubiesen actuado con mayor firmeza y cuidado, o denunciado a tiempo lo que pasaba con los Nule y los demás subcontratistas, especialmente en relación con los múltiples incumplimientos y el debilitamiento del músculo financiero de sus empresas, habrían evitado la catástrofe. En ese sentido, ampliar el régimen de responsabilidad de los asesores, consultores e interventores de contratos estatales en materia fiscal y disciplinaria, e imponer a los interventores un deber de información y de comunicación a las entidades públicas de los hechos o circunstancias que puedan constituir actos de corrupción tiene una relación notoria con el caso que nos ocupa.

2. La inhabilidad de contratistas que sean sancionados en la ejecución de contratos y por financiar campañas políticas. Las inhabilidades en la contratación pública están consagradas en los artículos 8 y 9 de la Ley 80 de 1993, complementados por el artículo 18 de la Ley 1150 de 2007 , en los que se estipulan las limitaciones a la capacidad para contratar con las entidades estatales por razones del interés público. ${ }^{11}$

11 Para mayor detalle véase: Corte Constitucional. Sentencia C-489 de 1996. M. P.: Antonio Barrera Carbonell. 
El artículo 90 de la Ley 1474, que se refiere a las inhabilidades como consecuencia de la imposición de multas en la ejecución de contratos estatales, es una sanción al contratista que incumple reiteradamente con sus obligaciones contractuales. Los Nule incumplieron muchos de sus contratos y al no existir la figura de la inhabilidad por incumplimiento reiterado pudieron seguir licitando y contratando con el Estado. Esta inhabilidad está hecha para impedir que contratistas con incumplimientos reiterados ante distintas entidades estatales y a quienes se les impongan varias multas no puedan seguir con esas maniobras, y, tampoco, resultar adjudicatarios de nuevos contratos, como sucedió en el carrusel.

De igual forma, las normas sobre inhabilidades e incompatibilidades se quedaron cortas y por esa razón fue necesario inhabilitar a quienes financien campañas políticas (el artículo 2 de la Ley 1474 de 2011 adicionó el literal k al artículo 8 de la Ley 80 de 1993). En el carrusel, como se dijo anteriormente, aunque aún no ha sido confirmado, las investigaciones señalan que los contratistas implicados en el carrusel financiaron la campaña de Samuel Moreno a la Alcaldía de Bogotá. Si esta inhabilidad hubiese estado vigente, habrían estado inhabilitados para contratar durante la Alcaldía de Moreno.

Consagrar en el Estatuto Anticorrupción la inhabilidad para quienes hayan financiado de manera privada los partidos políticos fue un acierto, en la medida que contribuye a controlar esta práctica que se ha usado como método de corrupción continua, pues "quien recibe el fondo a nombre del partido se compromete a tomar una decisión o a influir para que otros tomen una decisión en el sentido querido del donante" (Malem Seña, 2002, pág. 114). Adicionalmente, para Carlos Fernando Galán, la financiación de campañas políticas y la falta de control sobre estas es la causa que permite el desarrollo de la corrupción.

Sin embargo, como lo relatan Lombana y Castro (2012), la inhabilidad fue restringida en el último debate en el Congreso a aportes superiores al dos punto cinco por ciento (2.5\%) de las sumas máximas a invertir por los candidatos en las campañas electorales, con lo cual se abre de nuevo la compuerta para que estas maniobras continúen.

3. Frente al manejo de anticipos en la contratación pública, el artículo 91 de la Ley 1474 lo regula de acuerdo con la tipología contractual o por el proceso de selección por el que se adjudica el contrato. Lo anterior está dirigido, si se revisan nuevamente los hechos del carrusel de la contratación, a confrontar las causas que permitieron esta situación: indebido manejo de anticipos en contratos de obra pública, concesión y construcción de redes de servicios públicos, los cuales son adjudicados por licitación pública. Adicionalmente, conforme al numeral 1 del artículo 2 de la Ley 1150 de 2007, debe tenerse en cuenta que por este proceso se adjudican los contratos de mayor cuantía, es decir, aquellos en los que están en juego los mayores recursos del erario público.

Con esta norma el legislador buscó proteger la adecuada inversión de los anticipos a través de 
contratos de fiducia pública, para que personas como los Nule no puedan invertirlos para pagar las deudas de otro contrato y apalancarse sucesivamente. Los contratos de fiducia pública estipulan que el dinero aportado por las entidades contratantes proveniente del anticipo, solo puede invertirse en la ejecución del contrato específico, con lo cual la disponibilidad presupuestal desembolsada por el Estado no podrá ser usada en la ejecución de un contrato diferente.

4. Entre las medidas penales que se consagraron en la Ley 1474, están: la exclusión de beneficios en los delitos cometidos contra la administración pública, el aumento del término de prescripción y la creación del delito por acuerdos restrictivos de competencia, todas dirigidas a disuadir a los corruptos. ${ }^{12}$

Por estas razones, es posible concluir que ciertas normas del Estatuto Anticorrupción no solo fueron una reacción del Estado colombiano a los hechos del carrusel de la contratación y, por tanto, provocadas por un caso específico, sino que corresponden a una supuesta metodología de prevención. Desafortunadamente, la reacción del Estado fue posterior a los hechos, dejando al descubierto las fallas de las instituciones que permitieron que esto sucediera. Solo hasta el año 2013, como se verá más adelante, se inició la formulación de una política pública realmente dirigida a la prevención. Hasta este año, por fin, el Estado se dio cuenta que en ma-

12 Aunque no es el tema principal de este ensayo, se resalta que el aumento de las penas obedece a una visión correccionalista de los criminales y al interés del Estado de imponer castigos acordes con las conductas ilegales, con el fin de legitimarse frente a la sociedad y mantener la coerción estatal (Garland, 1997). teria de control sobre el manejo de los recursos públicos las reacciones legislativas posteriores tienen más de populismo legislativo que de control definitivo.

\section{B. Análisis de la efectividad del Estatuto Anticorrupción en la percepción de la corrupción y de la confianza del derecho como instrumento de cambio social, además de la nueva estrategia de política pública anticorrupción del Gobierno apoyada en el Plan Nacional de Desarrollo}

En este aparte la idea básica que se va a desarrollar es la siguiente: la promulgación del Estatuto Anticorrupción evidencia la falsa premisa de la confianza depositada en el derecho positivo como instrumento para combatir de manera eficiente la corrupción, y obedece a un momento histórico determinado que exigía resolver los conflictos generados por el carrusel de la contratación. Adicionalmente, ese cambio normativo permite mostrar que la discusión sobre la corrupción no se agota en las medidas legislativas, y que el Gobierno solo hasta el 2013 se dio cuenta de esto y se apoyó en la Ley 1450 para desarrollar una nueva política pública diseñada en función de la prevención.

Para tratar esta idea se analiza la eficacia de la norma en función de su aceptación social, y los efectos que ha logrado frente al cambio cultural. Luego se revisa cómo la discusión sobre corrupción no puede ser atendida únicamente desde planteamientos jurídico-legislativos, sino que debe considerarse también desde una óptica de 
evaluación del incumplimiento de las normas y la desconfianza en las instituciones.

La teoría jurídica clásica ha señalado que una norma jurídica es válida si es expedida por la autoridad competente y no contraría ninguna norma superior, esto es, su validez formal ${ }^{13}$ y material ${ }^{14}$. Sin embargo, los sociólogos y realistas jurídicos han decidido analizar el derecho desde la eficacia de las normas. En este artículo se toma la eficacia como punto de análisis, esto es, la aceptación social y la efectividad de la norma para atender el problema por el que surge. Esto significa que "las normas son, por tanto, valores que buscan la superación de dichos problemas. Pero obviamente no son valores abstractos, sino valores en concreto" (Giraldo Ángel et al., 2010, pág. 93).

En las discusiones entre juristas se ha contrapuesto o asimilado a un mismo concepto la eficacia -entendiéndola en sentido amplio- y la validez, sin embargo, en este texto deben entenderse como dos conceptos diferentes pero con una estrecha relación entre sí, por cuanto la validez material supone la legitimidad de la norma y la eficacia se ocupa de las consecuencias sociales de esta. ${ }^{15}$ Así, una norma eficaz será

13 Esto es, que la norma fue expedida por la autoridad facultada y conforme al procedimiento establecido por el sistema jurídico (Mendoza Rojas, 2011).

14 Significa que el contenido de la norma se encuentra acorde con los lineamientos establecidos por las normas de superior jerarquía (Mendoza Rojas, 2011).

15 El realismo jurídico apoya la tesis según la cual, la validez de una norma es lo mismo que su eficacia. Uno de sus mayores exponentes fue Alf Ross. Por el contrario, para el positivismo jurídico la eficacia es una condición de validez o de existencia de una norma jurídica; su mayor exponente fue Hans Kelsen (Galvis, 2011). aquella que es acatada y que desmuestra resultados fehacientes de su cumplimiento y aceptación social, lo cual además la legitima.

Así, lo que procede a continuación es evaluar la eficacia del Estatuto Anticorrupción desde su aceptación social, teniendo como base el índice de percepción de la corrupción (IPC) construido por la organización no gubernamental (ONG) Transparencia Internacional, para lo cual se usó como parámetro los años 2008 a 2013. Este índice "establece cada año un ranking de países basado en los niveles de corrupción en el sector público percibidos por empresarios, analistas de riesgo y ciudadanos comunes" (LatinoaméricaLibre.org, 2011). Todos los años esta oNG evalúa la percepción de los niveles de corrupción de 180 países aproximadamente, de los cuales 32 son de América. ${ }^{16}$ Este indicador se escogió por su aceptación social y gubernamental y, además, por provenir de una de las organizaciones anticorrupción con mayor credibilidad internacional.

16 La diferencia entre el Barómetro y el IPC consiste en que el primero "es una encuesta de opinión pública que permite conocer las perspectivas del público en general sobre la corrupción y el impacto que esta tiene en sus vidas, incluida las experiencias personales de soborno. [...] EI IPC refleja la percepción de observadores informados acerca de la corrupción en el sector público y la política" (Vásquez Caro, 1994). 
Los resultados que Colombia ha obtenido en los últimos seis años en el IPC son los siguientes ${ }^{17}$ :

\begin{tabular}{|c|c|c|}
\hline AÑO & $\begin{array}{c}\text { PUESTO } \\
\text { A NIVEL } \\
\text { MUNDIAL }\end{array}$ & $\begin{array}{c}\text { PUNTAJE (donde 1 es para } \\
\text { nada corrupto } \mathbf{5} \text { es muy } \\
\text { corrupto) }\end{array}$ \\
\hline 2008 & 70 & 3,8 \\
\hline 2009 & 75 & 3,7 \\
\hline 2010 & 78 & 3,5 \\
\hline 2011 & 80 & 3,4 \\
\hline 2012 & 94 & $36^{18}$ \\
\hline 2013 & 94 & 36 \\
\hline
\end{tabular}

La anterior relación de resultados hace notorio el aumento en la percepción de corrupción que tienen la ciudadanía, los empresarios y los analistas de riesgo. En cuatro años Colombia cayó 24 posiciones en el ranking de países y la percepción de la corrupción creció a pesar de la reacción del Estado. ${ }^{19}$ Entre 2012 y 2013 no hubo ninguna variación en el puntaje; se mantuvo en el puesto 94.

Otra conclusión a la que se puede llegar con estos resultados del IPC es que la percepción de corrupción venía disminuyendo desde el año 2008, y con la promulgación de las leyes 1450 y

17 Para consultar los resultados visítese http://www.transparency.org/ research/cpi/overview

18 Desde el 2012 cambió la metodología de medición y ahora, como reflejo de esto, el índice se presenta en una escala donde 0 es mayor corrupción y 100 es menor corrupción.

19 Hay quienes afirman que la percepción puede variar por el aumento de las acciones del Estado y la visibilidad de nuevos casos, sin embargo, para Daniel Kaufmann, director de gobernabilidad del Instituto del Banco Mundial, este nuevo enfoque en las mediciones de percepción "se concentran en crear indicadores basados en encuestas de expertos y en medir, a través de encuestas de empresas y usuarios de servicios públicos, la magnitud de la mala gestión y el soborno" (Banco Mundial, 2004). Además, puede haber otros indicadores que también demuestran los altos niveles de corrupción, por ejemplo, el índice Golden y Picci (GyP).
1474 de 2011 no solo mantuvo esta tendencia, sino que adquirió un ritmo más rápido, pues Colombia pasó del puesto 80 al 94 entre 2011 y 2012. Incluso, el aumento de las medidas penales y el castigo no logró disuadir a los corruptos, como parece demostrarlo el IPC. De acuerdo con esto se puede concluir que el esfuerzo del legislador, hasta ese momento, no incidió en la realidad social y los ciudadanos del común no han visto que el Estatuto Anticorrupción sea eficaz.

Sin embargo, Rafael Merchán, actual secretario de Transparencia de la Presidencia de la República, como vocero del Gobierno afirma que la Ley 1474 ha servido para contrarrestar y controlar los casos de corrupción y "sí ha sido una buena herramienta" (Merchán, 2013).

Tomando como base las variaciones del IPC, se puede argumentar que el Estatuto Anticorrupción carece de eficacia material, pues su promulgación y puesta en acción no ha incidido en la percepción de la corrupción, toda vez que dicho índice en vez de disminuir ha aumentado. En consecuencia, la visión del Gobierno podría encajarse dentro de la eficacia simbólica, esto es, un concepto que el derecho ha traído de la antropología y de la sociología, por medio del cual se explica la distancia existente entre la validez de la norma y la eficacia real de esta. En palabras de García Villegas (1989):

los contratiempos que surgen en la etapa ejecutiva conducen con frecuencia a la ineficiencia de las normas; suele entonces atribuirse la responsabilidad de este fracaso, a la falta de previsión de la realidad por parte del creador del texto jurídico o a la incapacidad de las ins- 
tancias encargadas de la aplicación para llevar a buen término los objetivos propuestos en la norma, o incluso a la falta de comunicación entre ambas instancias (pág. 37).

Como se desprende de los resultados analizados anteriormente, el Estatuto Anticorrupción no ha tenido hasta el momento eficacia, y demuestra que la ley por sí sola no es un instrumento suficiente para combatir un fenómeno social tan complejo como es la corrupción. Sencillamente, es un instrumento que colabora con la persecución de unos fines, pero que en sí mismo no contiene el fundamento para lograr el cambio cultural que el país necesita, por lo que el síndrome legislativo no va a acabar con el flagelo de la corrupción. Creer que el cambio social se puede dar por medio de la ley es caer en el fetichismo legal y engañarse con la aprobación de la ley. Para Julieta Lemaitre (2009),

el fetichista tiene la absurda creencia de que el derecho cambia la realidad social. En ese último sentido, el fetichismo legal se refiere a no darse cuenta del abismo que separa la aprobación de una ley, de su aplicación, y estar de alguna manera engañado por el ritualismo de la norma, posponiendo indefinidamente la confrontación con sus problemas de aplicación (pág. 384).

Según Maldonado Copello, el Estado colombiano sí se preocupó y cayó en el fetichismo legal creyendo que la expedición de normas jurídicas iba a frenar el flagelo de la corrupción. Así mismo, recalcó la necesidad de generar una política pública aliada al recién promulgado Estatuto Anticorrupción. En sus palabras: "Parecería que el Gobierno actual le da más peso a la expedición de normas contra la corrupción que a la creación de condiciones institucionales para su efectiva aplicación. De ahí la necesidad de articular el proyecto de ley anti-corrupción a una política integral de lucha contra la corrupción (2011, pág. 1).

Como bien lo dice este autor, las leyes deben acompañarse de una política integral, lo que ha intentado el Gobierno a través de documentos como el Conpes 3654 del 12 de abril de 2010, en el cual señala que la rendición de cuentas es una expresión de control social que busca la transparencia de la gestión de la administración pública. Sin embargo, esto había sido dejado a un lado hasta que en el 2013 el Gobierno decidió comenzar la recuperación de las políticas públicas de prevención contra la corrupción.

En 1999, Martha Elena Badel Rueda ya anunciaba al Gobierno las posibles causas de la corrupción y llamaba su atención en los efectos económicos que sobrevendrían si no se implementaban políticas públicas serias para su control. Dentro de las causas posibles enumeró: 1. la ineficiencia del Estado; 2. Ia discrecionalidad de los funcionarios públicos generada por su deficiencia en la toma de decisiones y su falta de compromiso; 3. el monopolio de los servicios que se prestan y la falta de visión innovadora resultante de la carencia de competidores; 4. la falta de desincentivos a la corrupción que se representa en la posibilidad de adquirir grandes cantidades de dinero; 5. la baja probabilidad de ser descubiertos o lo que es lo mismo, una mala calidad del control; 6 . los bajos castigos para las 
actividades corruptas; y 7. la baja sanción social para los individuos corruptos.

Además de Badel Rueda varios autores han expuesto las posibles causas de la corrupción, pero ¿el Estado colombiano desde qué óptica aborda la discusión de este problema? Como lo mencionó Rafael Merchán, el Estado había abordado la corrupción como un tema normativo y de eficiencia de las instituciones, para lo cual el Estatuto Anticorrupción ha sido una buena herramienta. Sin embargo, hoy en día está modificando su método de acción y se está dando cuenta que la "corrupción es un tema subdiagnosticado porque avanza más rápido que lo que avanzan las instituciones" (2013). El Gobierno se apoyó en el Plan Nacional de Desarrollo 2010-2014, que tenía olvidado en lo referente a la corrupción, y comprendió que esta debía prevenirse en vez de hacer un control posterior, por lo que, como menciona Merchán, ahora se va a abordar como un tema de política pública en vez de una necesidad de transformación normativa. Para eso, está en desarrollo un documento del Consejo Nacional de Política Económica y Social (Conpes) donde se plantea la política pública anticorrupción del país y se diseñaron 110 acciones para 22 entidades.

Según lo anterior, aunque en el Plan Nacional de Desarrollo 2010-2014, que fue aprobado en el 2011, se incluyó el aparte Buen gobierno, participación ciudadana y lucha contra la corrupción, solo hasta el 2013 el Gobierno decidió ejecutar esta idea y formular la política pública necesaria para atender este flagelo desde otra perspectiva. El Estado colombiano había limita- do sus esfuerzos a la óptica jurídica, restringiendo el campo de acción y desconociendo que "la corrupción puede consistir en prácticas esporádicas o en formas sistémicas, bajo la figura de la captura del Estado y especialmente lo que algunos autores denominan la reconfiguración cooptada del Estado, fenómeno en el cual se produce una captura sistemática de las instituciones estatales no solamente con fines económicos" (Maldonado Copello, 2011). La corrupción es un tema que no se agota en el derecho, sino que debe ser analizado desde otras áreas del conocimiento, y con base en ellas debe complementarse el derecho de tal forma que por su rigidez no se quede corto frente a los cambios y adaptaciones de la corrupción. Esas áreas pueden ser la economía, ${ }^{20}$ la antropología, ${ }^{21}$ la sociología, ${ }^{22}$ entre otras.

Con base en las normas analizadas en este texto, las disposiciones de contratación estatal consagradas en el Estatuto Anticorrupción parecen pensadas y diseñadas para determinar cómo se incumplió y no para identificar por qué se incumple y anticipar los correctivos. Esta diferenciación es importante tenerla presente porque la legislación sobre casos particulares no permite desligar el incumplimiento de otros

20 Véase: Leff (1964) y Huntington (1968), quienes analizaron la relación positiva entre corrupción y crecimiento económico. En Vergara Gamarra (2006).

21 Por ejemplo: una interpretación antropológica de la corrupción parte de la premisa que las definiciones normativas y legales son demasiado limitadas para explicar el fenómeno, pues desde esta área del conocimiento es una práctica social compleja (Huber, 2005).

22 Para esta disciplina el fenómeno de la corrupción puede y debe ser tratado sociológicamente, pues es una acción social con un mínimo de bilateralidad social: su causa y sus efectos son sociales (Prado Olivera, 1997). 
actores sociales y su efectividad material disminuye. Por el contrario, si la regulación se analiza desde el por qué se incumple, la norma jurídica será más propicia para erradicar la fuente del problema, aumentar su eficacia material y responder al contexto jurídico social.

La lucha contra la corrupción como lineamiento estratégico de buen gobierno, contenido en la Ley 1450, mostró la visión del presidente Santos en materia de transparencia, pero fueron necesarios más de tres años de su Gobierno para que fuera puesta en acción.

El Estado colombiano dejó pasar décadas enteras sin comprender este fenómeno, atendiéndolo desde una óptica normativa, y como consecuencia de ello muchos billones de pesos que podrían haberse invertido en educación, salud, primera infancia, infraestructura, trabajo y muchos otros factores necesarios para el desarrollo del país se perdieron en manos de los corruptos.

\section{CONCLUSIONES}

El carrusel de la contratación es un caso de corrupción tan grande que aún las autoridades no han logrado descifrarlo en su totalidad, y en este texto se hizo una exposición muy breve de Ios hechos más relevantes. Sin embargo, es claro que esta situación se dio con la actuación de contratistas y funcionarios públicos de alto rango nacional y llegó a un nivel de descaro tal que se apropió el Estado, mientras las autoridades, en teoría, desconocían estos hechos. Gracias a las investigaciones del exconcejal (hoy senador)
Carlos Fernando Galán y de su equipo se prendieron las alarmas, y con el apoyo de otros concejales y de los medios de comunicación se hizo público el que hasta el momento es uno de los casos más graves en materia de corrupción que haya vivido el país.

A raíz de este escándalo, el Estado colombiano reaccionó con la promulgación de la Ley 1474 de 2011 (Estatuto Anticorrupción), aunque hasta el momento, a la luz del índice de percepción de corrupción de Transparencia Internacional, esta no ha sido eficaz. Además, con esta reacción el Estado demostró que algunos artículos de dicho Estatuto fueron pensados para un caso específico y su debilidad en la formulación de políticas públicas diseñadas para la prevención de estos actos delictivos.

Adicionalmente, aunque desde la visión del Gobierno el Estatuto Anticorrupción parece tener eficacia simbólica, se puede concluir que el derecho por sí solo no es un instrumento suficiente para combatir un fenómeno social tan complejo como la corrupción. El Estado debe apoyarse en otras áreas del conocimiento que le permitan comprender su complejidad, y dirigir la lucha contra este flagelo de manera integral de modo que se avance en una cultura de transparencia, se reduzcan los altos índices de percepción de corrupción y, más importante aún, se frenen los casos de apropiación indebida de los recursos públicos.

El carrusel de la contratación y las reacciones del Estado evidencian que fueron necesarios más de tres años del Gobierno Santos para 
poner en acción una política pública diseñada para prevenir la corrupción como eje fundamental, en vez de centrarse en el control posterior. Esta le permitirá al Gobierno mayor agilidad en la lucha y atacar los intentos de mimetización de los corruptos.

Finalmente, el lector debe tener en cuenta que la corrupción es el mayor obstáculo para el avance de un país porque frena la inversión y el desarrollo social (Consejo Privado de Competitividad, 2013). Por eso, es importante que la lucha contra la corrupción no se vea como un esfuerzo que compete solo al Gobierno, sino que todas las fuerzas vivas del país se unan a este propósito e interactúen sancionando socialmente este flagelo, pues “[...] la adopción de leyes no resulta eficiente si no hay una cultura que rechace el fenómeno [de la corrupción] y no se toman acciones concretas de defensa" (Vélez, 2013).

\section{Referencias}

Amarribo. (2010). El combate a la corrupción en las alcaldías de Brasil. (4 $4^{\mathrm{a}}$ ed.). Avina (Trad.). São Paulo: 24x7 cultural Ltda.

Badel Rueda, M. E. (1999). Costos de la corrupción en Colombia. Recuperado de https:// www.dnp.gov.co/portals/0/archivos/documentos/dee/archivos_economia/111.pdf

Banco Interamericano de Desarrollo. (2012). Gobierno abierto y transparencia focalizada. Tendencias y desafíos para América Latina y el Caribe. N. Dassen y J. C. Vieyra (edits.). Washington: BID.

Banco Mundial. (8 de abril de 2004). La revolución de los datos: cómo medir la gobernabilidad y la corrupción. Recuperado el 13 de noviembre de 2013, de http://www.bancomundial.org/temas/anticorrupcion/medir.htm

BRC Investor Services S. A. (03 de mayo de 2011). Primera emisión de Bonos Ordinarios Fideicomiso 3-4-1318.

Caracol Radio. (25 de febrero de 2011). Recuperado de http://www.caracol.com.co/ noticias/judicial/cronologia-del-carruselde-contratacion-en-bogota/20110225/ nota/1431573.aspx

Consejo de Estado. Sala de lo Contencioso Administrativo. Sec. Quinta. C. P: Susana Buitrago Valencia. 25 de enero de 2013. Radicado n. ${ }^{\circ}$ 11001-03-15-000-2012-02311-00.

Consejo Privado de Competitividad. (2013). Informe nacional de competitividad 20122013. Recuperado de http://www.compite. com.co/site/wp-content/uploads/2012/11/ INC-2012-2013.pdf

Convención de las Naciones Unidas contra la Corrupción. (s. f.). Preámbulo. Recuperado de http://www.unodc.org/pdf/corruption/ publications_unodc_convention-s.pdf

Departamento Nacional de Planeación. (2010) ¿Qué es el Plan Nacional de Desarrollo? Re- 
cuperado de http://www.dnp.gov.co/pnd. aspx

Departamento Nacional de Planeación. (2010). Respuesta a la consulta número 2010-663004907-2.

Departamento Nacional de Planeación. (2010). Plan de Desarrollo 2010-2014. Resumen ejecutivo. Recuperado de https://www. dnp.gov.co/LinkClick.aspx?fileticket $=4-J 9 V$ FE2pl\%3D\&tabid=1238

El Tiempo. (2010). Invías. Recuperado de www. eltiempo.com/justicia/ARCHIVO/ARCHIvo-9182120-0.xls

El Tiempo. (2011). La caída del imperio de los Nule. Recuperado de http://www.eltiempo. com/Multimedia/infografia/nule/

Galán, C. F. (12 de noviembre de 2013). El carrusel de la contratación. Entrevista de D. Escallón. [Cinta de audio].

Galvis, O. (2011). La fuerza fáctica de lo normativo: relaciones entre la validez y la eficacia. En P. Moncada Roa, Introducción al derecho: obra en construcción (págs. 315-366). Bogotá: Temis.

García Villegas, M. (1989). El derecho como instrumento de cambio social. Revista Facultad de Derecho y Ciencias Políticas: Universidad Pontificia Bolivariana, 29-44.
Garland, D. (1997). Crimen y castigo en la modernidad tardía. Bogotá: Siglo del Hombre Editores.

Giraldo Ángel, J., Giraldo, M., y Giraldo, A. (2010). Metodología y técnica de la investigación sociojurídica. (4 ${ }^{\mathrm{a}}$ ed.). Bogotá: Librería Ediciones del Profesional Ltda.

Huber, L. (2005). Una interpretación antropológica de la corrupción. Recuperado de http:// www.cies.org.pe/files/documents/investigaciones/descentralizacion/una-interpretacion-antropologica-de-la-corrupcion.pdf

Instituto de Ciencia Política Hernán Echavarría Olózaga. (Septiembre de 2011). Boletín de Seguimiento Legislativo - Estatuto Anticorrupción. Recuperado de http://www.icpcolombia.org/archivos/observatorio/boletin_188

Kelsen, H. (2009). Teoría pura del derecho, introducción a la ciencia del derecho (16 ${ }^{\mathrm{a}}$ ed.). R. J. Vernengo (Trad.) México: Porrúa.

LatinoamericaLibre.org. (2011). Índice de percepción de la corrupción. Recuperado de http://www.latinoamericalibre.org/indices/ indice-de-percepcion-de-la-corrupcion-ipc/

Lemaitre, J. (2009). El derecho como conjuro: fetichismo legal, violencia y movimientos sociales. Bogotá: Siglo del Hombre Editores.

Lombana, J. y Castro, C. G. (2012). Estatuto anticorrupción y política criminal. En F. Sintura Varela y N. Rodríguez, El Estado de Derecho 
colombiano frente a la corrupción (págs. 149-179). Bogotá D. C.: Universidad del Rosario.

López Medina, D. E. (2008). La letra y el espíritu de la ley: reflexiones pragmáticas sobre el lenguaje del derecho y sus métodos de interpretación. Bogotá: Editorial Temis.

Maldonado Copello, A. (2011). La lucha contra la corrupción en Colombia: la carencia de una política integral. Recuperado de http://library.fes.de/pdf-files/bueros/kolumbien/08285.pdf.

Malem Seña, J. F. (2002). La corrupción. Aspectos éticos, económicos, políticos y jurídicos. Barcelona: Gedisa.

Medina, A. M. (23 de junio de 2010). [Carta enviada al Instituto Nacional de Concesiones -Inco-]. Ref. Autopista Bogotá Girardot S. A. Oficio 0414-01.

Mejía, D. (31 de octubre de 2013). El carrusel de la contratación. Entrevista de D. Escallón.

Mendoza Rojas, F. (2011). Validez y vigencia temporal: avatares de una norma errante. En P. Moncada Roa, Introducción al derecho: obra en construcción (págs. 71-126). Bogotá: Temis.

Merchán, R. (30 de octubre de 2013). Estatuto Anticorrupción. Entrevista de D. Escallón.

Ministerio del Interior y de Justicia. (Junio de 2011). Presentación del Estatuto Anticorrup- ción. Recuperado de http://www.legis.com. co/BancoMedios/Documentos\%20PDF/cartillaestatutoanticorrupcion.pdf

Pieth, M. (2011). Harmonising Anti-Corruption Compliance: The OECD Good Practice Guidance 2010. Zurich: Dike.

Programa de las Naciones Unidas para el Desarrollo. (2011). Buenas prácticas de transparencia en la gestión contractual. Bogotá: PNUD.

Prado Olivera, M. (1997). Hacia una sociología de la corrupción. Recuperado de http://sisbib.unmsm.edu.pe/bibvirtual/publicaciones/alma_mater/1999_n17/socio_corrup. htm

Revista Semana. (11 de septiembre de 2010). La caída del grupo Nule. Recuperado de http://www.semana.com/nacion/articulo/ la-caida-del-grupo-nule/121790-3

Revista Semana. (06 de octubre de 2012). "Carrusel de la contratación" en Bogotá: se destapó Emilio Tapia. Recuperado de http:// www.semana.com/nacion/articulo/carrusel-contratacion-bogota-destapo-emilio-tapia/265981-3

Revista Semana. (11 de octubre de 2012). Emilio Tapia revela "el mapa de la corrupción". Recuperado de http://www.semana.com/ nacion/articulo/emilio-tapia-revela-mapacorrupcion/266184-3 
Revista Semana. (13 de abril de 2013). El segundo bogotazo. Recuperado de http://www. semana.com/nacion/articulo/el-segundobogotazo/339828-3

Superintendencia de Sociedades. (10 de septiembre de 2010). Liquidación judicial. Recuperado de http://www.supersociedades.gov.co/web/documentos/AUTO\%20 LIQUIDACI\%C3\%93N\%20MNV\%20SA.pdf

Transparencia Internacional. (2009). Barómetro global de corrupción. Recuperado de http://www.transparencia.org.es/ BAR\%C3\%93METRO\%2 OGLOBAL\%20 2009/Informe_detallado_sobre_el_ Bar\%C3\%B3metro_Global_2009.pdf
Vásquez Caro, J. (1994). Corrupción en América Latina: un peso muerto de ineficiencia e inestabilidad en gobernabilidad y reforma del estado. Bogotá: Editores Cárdenas Santamaría.

Vélez, J. (2013). Transparencia en los procesos de contratación: un compromiso de todos. Bogotá.

Vergara Gamarra, J. R. (marzo de 2006). Pobreza, corrupción y participación política: una revisión para el caso colombiano. Recuperado de http://www.banrep.gov.co/ sites/default/files/publicaciones/archivos/ DTSER-70_(VE).pdf 